\title{
The Physics of Convection in Massive Stars
}

\author{
Casey A. Meakin ${ }^{1,2,3}$ \\ ${ }^{1}$ New Mexico Consortium, Los Alamos, NM 87544 USA \\ ${ }^{2}$ Los Alamos National Laboratory, Los Alamos, NM 87545 USA \\ ${ }^{3}$ University of Arizona, Tucson, AZ 85721 USA \\ email: casey.meakin@gmail.com
}

\begin{abstract}
I summarize current state-of-the-art methods for treating the difficult problem of turbulent convection in stellar interiors and I discuss a powerful approach for analysis that allows one to leverage the most from 3D stellar models.
\end{abstract}

Keywords. convection, turbulence, stars: evolution, supernovae

\section{Introduction}

The shortcomings of turbulent mixing models for stars, including the mixing length theory of convection and various types of overshoot mixing prescriptions, are now widely acknowledged. Of particular concern are the errors related to accurately predicting the amount of mixing that takes place across convective boundaries which result in a wide range of modeling errors, including predicted nucleosynthetic yields, supernova progenitor structures, and stellar lifetime estimates, to name just a few. The problem of turbulent mixing is not confined to massive stars but impacts stars of nearly every mass. Therefore, one of the most pressing frontier questions in modern stellar structure and evolution is, how can we develop a better model of stellar turbulence? In this short article I briefly recount a promising line of inquiry into this problem that incorporates simulation modeling and classical turbulence analysis.

\section{RANS: A Powerful Tool for Investigating Stellar Turbulence}

As with any frontier science, defining terms and posing useful questions is an essential first step. And while research on the compressible, stratified, multi-species, reactive turbulence relevant to stellar interiors remains parked at the scientific frontier, much work has been done on simpler, related problems out of which has grown a powerful set of techniques for discussing the most important issues.

Of special importance is the method of Reynolds decomposition and the development of a set of statistical mean-field equations known as Reynolds-Averaged Navier-Stokes equations or RANS equations. The basic principle behind Reynold decomposition is to write a fluid property as the sum of its time-averaged and instantaneous-fluctuation components $\varphi(x, t)=\bar{\varphi}(x)+\varphi^{\prime}(x, t)$, where $x$ and $t$ are the space and temporal coordinates, respectively; the overbar denotes time (and sometimes also space) averaging; and the prime indicates the time dependent, fluctuating component of the field. RANS equations are then developed by time or space averaging the conservation laws after having rewritten the fluid properties in terms of Reynolds decomposed fields; and a complementary set of moment equations are developed by multiplying the conservation equations by products of fluctuating components prior to applying the averaging operator. This procedure is the workhorse for turbulence research, and remains one of the primary windows into 
the nature of turbulent flow and has been elaborated upon in standard texts written over the last several decades (e.g. Landau \& Lifshitz 1959; Hinze 1959; Tennekes \& Lumley 1972; Frisch 1995; Pope 2000; Chassaing 2002).

The RANS equations developed in this way both define a set of turbulence quantities precisely (such as turbulent kinetic energy, turbulent stress, and energy flux), and include a set of time dependent equations describing the evolution of these quantities and their relationships. But as excellent a result as this is, Mother Nature does not give up her secrets so easily. The nonlinear terms in the conservation laws ultimately result in an infinite set of coupled equations wherein the evolution for each statistical moment depends on moments of a higher order, and we are faced with a closure problem akin to the BBGKY hierarchy in statistical physics. The research problem is to find a way to truncate the set of equations by modeling higher order terms as a function of lower order terms.

\section{Confronting the Closure Problem}

While the RANS equations offer a precise way to discuss and analyze turbulent systems, we are ultimately left with an incomplete description whenever we truncate our system of equations to a finite, and therefore usable, set of statistical moments. Therefore, in order to develop a useful model we must find a way to close the system. In physical terms, this means that we need to develop a closed form description of how fluctuations correlate with each other.

The mixing length theory (MLT) is in fact a closure model: it prescribes, albeit in a simplistic fashion, how velocity and temperature fluctuations are correlated. MLT, then, allows us to close the mean field equations of stellar structure and evolution and find solutions. More sophisticated models, such as those developed by Kuhfuss (1986) and Canuto (1992), have also been produced over the years and draw on a more detailed picture of how turbulence behaves. However, in all cases, it remains unclear which model is best suited to treating stellar turbulence.

Without strong justification for one model over the next, it makes sense to keep on using the most user-friendly available, which remains MLT. Today, however, we are starting to witness a transformation in our ability to study turbulent flow that is being driven by computer simulations of ever finer granularity and that are now classifiable as representing truly turbulent flow.

\section{Leveraging 3D Simulation}

The beauty of the RANS approach to stellar turbulence is that it provides a well defined set of turbulence quantities and evolution equations that can be systemically investigated from a physical perspective. Combining this analytic tool with simulation data (or numerical experiments) of stellar convection is starting to provide justification for one type of turbulence model over another. Modern stellar simulation data (e.g. Meakin 2006; Arnett \& Meakin 2011) is of sufficient quality to begin testing the basic building-block assumptions that go into turbulence models, like MLT, thus providing a rational for choosing one over the next.

In Mocák et al. (2014), we have provided a "nuts and bolts" look at how this approach can be pursued. First, we have developed a set of RANS equations which are of particular interest for stellar evolution: those that are derived from the conservation laws of momentum, energy, and mass (including a multi-species compositional description) and are cast against a fixed (or slowly time varying) spherical background base state. Second, 
we have calculated a large collection of statistical mean fields from our diverse collection of 3D stellar simulation data, which includes a supernova progenitor model, as well as lower mass models including a helium-shell flash and a reg giant convective envelope.

As discussed in some detail in Meakin \& Arnett (2006, 2007a,b,c), Meakin (2006, 2008), Arnett et al. (2009), and Meakin et al. (2011), and then more explicitly in terms of RANS by Murphy \& Meakin (2011) and Viallet et al. (2013), this approach provides a path towards developing physical intuition on what might otherwise appear to be very elusive characteristics of turbulence, including the origin of composition and energy fluctuations, the mechanisms of transport, and the geometric structures underlying these flows. While the equations presented in Mocák et al. (2014) may appear similar to those recently published by Canuto (2011a,b,c,d,e), in other places we have adopted a different approach in our work. In particular, we present our equation set completely unmodeled and unclosed and without approximation. Our intention is to provide exact evolution equations together with what is in essence raw data to be used to check approximations used in models such as those presented in Canuto's and similar works. We have chosen this approach because we believe that the community's knowledge about turbulent mixing is still too limited to develop adequate and robust models. There is still much work to be done!

\section{Data Availability on the Web}

In an attempt to facilitate developments in this challenging field of study, we are striving to continue to make our simulation data available for download and use by as broad an audience as possible. At present the subset of data discussed in Viallet et al. (2013) is available at http://www.stellarmodels.org and includes as many as 113 mean fields for study. In addition, we have supplied a Python library for reading and plotting data together with a short sample script to get started working with our data.

\section{References}

Arnett, D., Meakin, C., \& Young, P. A. 2009, ApJ 690, 1715

Arnett, W. D. \& Meakin, C. 2011, ApJ 733, 78

Canuto, V. M. 1992, ApJ 392, 218

Canuto, V. M. 2011a, A\&A 528, A76

Canuto, V. M. 2011b, $A \mathscr{E} A$ 528, A77

Canuto, V. M. 2011c, A\&BA 528, A78

Canuto, V. M. 2011d, $A \mathscr{E} A$ 528, A79

Canuto, V. M. 2011e, A\&A 528, A80

Chassaing, P. 2002, Variable Density Fluid Turbulence, Springer Science and Business Media Frisch, U. 1995, Turbulence: The Legacy of A. N. Kolmogorov, Cambridge University Press

Hinze, J. O. 1959, Turbulence, McGraw-Hill

Kuhfuss, R. 1986, A\&A 160, 116

Landau, L. D. \& Lifshitz, E. M. 1959, Fluid Mechanics, Elesvier

Meakin, C. \& Arnett, D. 2007a, in F. Kupka, I. Roxburgh, \& K. L. Chan (eds.), IAU Symposium, Vol. 239 of IAU Symposium, pp 296-297

Meakin, C. A. 2006, Ph.D. thesis, The University of Arizona, Arizona, USA

Meakin, C. A. 2008, in L. Deng \& K. L. Chan (eds.), IAU Symposium, Vol. 252 of IAU Symposium, pp 439-449

Meakin, C. A. \& Arnett, D. 2006, ApJ (Letters) 637, L53

Meakin, C. A. \& Arnett, D. 2007b, ApJ 665, 690

Meakin, C. A. \& Arnett, D. 2007c, ApJ 667, 448

Meakin, C. A., Sukhbold, T., \& Arnett, W. D. 2011, Ap\&SS 336, 123 
Mocák, M., Meakin, C., Viallet, M., \& Arnett, D. 2014, ArXiv e-prints

Murphy, J. W. \& Meakin, C. 2011, ApJ 742, 74

Pope, S. B. 2000, Turbulent Flows, Cambridge University Press

Tennekes, H. \& Lumley, J. L. 1972, First Course in Turbulence, MIT Press

Viallet, M., Meakin, C., Arnett, D., \& Mocák, M. 2013, ApJ 769, 1

\section{Discussion}

AERTS: What would be the effect of internal gravity waves caused by a convective core on the internal rotation profile of a star, e.g. starting from a rigid $\Omega(r)$ profile: what would $\Omega(r)$ look like after a nuclear timescale?

MEAKIN: The mean field framework that I have described is well suited to study questions such as this, but there is still a lot of work to do to characterize the relevant fluid dynamics. It has become increasingly clear over the past several decades that there is not a "fundamental" theory of turbulence and mixing but that instead each instability and process needs to be paid special attention. Our $3 \mathrm{~d}$ simulation work to date has only included one case with a differential rotation profile and since it is representative of an advanced shell burning stage of evolution (oxygen burning) it is not relevant to main sequence evolution. Therefore, my own work does not speak directly to the question you ask. But I would add that the a definitive answer to your question will depend on both unspecified parameters, such as the absolute value of the initial rotational velocity profile, the mass of the star, it's composition, etc., as well as a variety of highly uncertain fluid dynamical processes such as mixing driven by double-diffusive instabilities and coreboundary interactions (e.g., "overshoot").

Puls: To make things even more complex: what about the radiation field (also for outer convective zones)?

MEAKIn: The character of radiative effects are very different between deep interior convection and surface convection. A diffusive treatment is sufficient to treat interiors while surface convection requires a more complete description of the radiation field, as you point out. A number of research groups have been simulating surface convection in 3D with great success. Our complimentary efforts have to date been focused instead on deep interior convection with an emphasis on the late burning stages in massive stars (post helium burning). Under these conditions, not only can radiation be treated very accurately as a diffusive process but the overall importance of the radiation field is significantly reduced due to the increased role played by neutrino emission which effectively takes over as the dominant "transport" process during these phases.

YASUTAKE: What is the role of rotation in 3D simulations? Is it important or not? If important, we need $3 \mathrm{D}$ to $2 \mathrm{D}$ applications for stellar evolution instead of $3 \mathrm{D}$ to $2 \mathrm{D}$ simulations. Is it correct?

MEAKIN: From the point of view of 3D simulation, rotation is simply treated as an azimuthal velocity field. Whether it is important or not depends on the strength of the rotation and its gradients as is usually the case. As you correctly point out, a selfconsistent treatment of the mean velocity field for a star that possesses dynamically significant angular momentum requires at least a $2 \mathrm{D}$ description. In some cases, 3D might be required. 
YASUTAKE: Can you calculate the front of combustion in your 3D HD simulation? You showed the effects of resolution. I am afraid you did not check the convergence of scale. In my opinion we need AMR technique for hydrodynamic simulation including nuclear burning front, right?

MEAKIN: The nuclear burning that occurs during the late burning stages in a massive star is not characterised by a thin flame front like the deflagrations that takes place in Type Ia supernovae explosions, so resolving the "front" is not a challenge. Nevertheless, the question of numerical resolution is still an important one for stellar convection simulations and this remains an active area of study. Our resolution studies, together with some basic fluid dynamical arguments, give us confidence that we are resolving the important processes within the convective layers that we are simulating (see, e.g., Section 4.7 of Viallet et al. 2014). The convective boundaries, on the other hand, have been more difficult to understand. Whether or not we have numerically converged mixing rates at these boundaries remains an open question and we are indeed beginning to study this part of the problem with AMR.

NoELs: You showed us how important it was to go from simple MLT to more realistic 3D models of convection. Would it be possible to come back from there towards an "adjusted" MLT with "adjusted" convection criteria? This would be very convenient to deal with stability analysis.

MEAKIN: It is important to be able to construct simplified models for whatever hydrodynamic processes we discover in our 3D simulations. Whether or not it is possible to incorporate all of the important physics as modifications to the current generation of MLT-based 1D models remains an open question. It is possible that an implementation based on a Reynolds-Averaged Navier-Stokes (RANS) approach is required and I would argue that there are already signs that we are starting to see the limitations of the current generation of codes, even those with "embellished" MLT style models. It is only through hard work and experience that we will be able to answer this question with certainty so we need to keep pushing ahead. 